\title{
MS23-P04 | Will PdPB CONFIRM THE PREDICTION THAT ITS STRUCTURE IS MOdULATED?
}

Folkers, Laura (Centre for Analysis and Synthesis, Lunds Universitet, Lund, SWE); Lidin, Sven (Lund University, Lund, SWE)

Recently, crystals of $\mathrm{PdPb}$ were successfully grown. Preliminary room temperature data shows a triclinic modulated structure with a q-vector $(1 / 20.2451 / 2)$ similar to that of Auln at room temperature.

The structure of Auln was elucidated not long ago, showing its structural changes with temperature [1]. Interest in Auln grew since it proved harder to grow single crystals from this material than expected and since its differential scanning calorimetry (DSC) data shows one solidification peak but two melting peaks. With the DSC data at hand, growth of a single crystal was possible and temperature dependent diffraction data could be obtained. This data revealed that Auln, believed to be of the TII type [2], actually only shows the Tll structure above the temperature of $443^{\circ} \mathrm{C}$ and at lower temperatures undergoes a Peierls type like distortion before becoming incommensurate [1].

$\mathrm{PdBi}$ emerges to be a similar case, with atomic positions of the reported high temperature phase structure corresponding to the TII type structure [3]. Also the DSC measurement shows the same behaviour as for Auln. As expected, a synchrotron study of $\mathrm{PdBi}$ shows that the structure transforms from a commensurate super structure, via an incommensurate structure, to a disordered structure between room temperature and $300^{\circ} \mathrm{C}$.

[1] Folkers, Simonov, Wang, Lidin, InorgChem., 57, (2018), 2791.

[2] Schubert, Rösler, Kluge, Anderko, Härle, Naturwissenschaften, 40, (1953), 34

[3] Zhuravlev, Zhurnal Eksperimental'noi i Teoreticheskoi Fiziki', 5, (1957), 1064. 